\title{
Simulation of a Four-stage Depressed Collector for a W-band Gyro-BWO
}

\author{
Liang Zhang, Wenlong He, Adrian W. Cross, Alan D. R. Phelps, Craig Donaldson and Kevin Ronald \\ SUPA, Department of Physics, University of Strathclyde, Glasgow, G4 0NG, Scotland, UK
}

\begin{abstract}
To improve the overall efficiency of the W-band gyrotron backward wave oscillator (gyro-BWO) currently been built in university of Strathclyde, an energy recovery system using a four-stage depressed collector was simulated and designed. The spent beam information was exported from the simulation of the gyro-BWO using 3D PIC code MAGIC. The geometry of the depressed collector was optimized using a genetic algorithm to achieve the optimum overall recovery efficiency for specific parameters of the spent beam. Secondary electron emissions were simulated to investigate the effects of the secondary electrons on the overall recovery efficiency and the backstreaming of the electrons from the collector region.
\end{abstract}

\section{INTRODUCTION AND BACKGROUND}

$\mathrm{G}$ YRO-BWOS are gyrodevices based on the electron cyclotron maser instability. Their main advantage is the wide frequency tuneability and high power capability in the millimeter and sub millimeter range. Research of gyro-BWOs based on a helically corrugated waveguide demonstrated excellent results in achieving high-power and wide frequency tuneability [1,2]. Simulation results of the W-band Gyro-BWO currently being built at the University of Strathclyde would have a $3 \mathrm{~dB}$ frequency bandwidth of $\sim 84-104 \mathrm{GHz}$ at $17 \%$ interaction efficiency and $10 \mathrm{~kW} \mathrm{CW}$ power [3]. To increase the overall efficiency of this device, an energy recovery system using multi-stage depressed collectors to recover the energy from the spent beam that is lost in processes such as heating of the walls of the collection region was designed and is presented in this paper.

The basic diagram of the W-band gyro-BWO experimental setup is shown in Fig. 1. A cusp electron beam [4-6] is used to drive the beam-wave interaction in a helical corrugated interaction region. A Bragg reflector acting as a microwave short at the end of the helically waveguide, can be used to reflect the radiation back to the system; the backward propagating microwaves can then be extracted using a waveguide coupler. The spent electrons propagating in the opposite forward direction, on exiting the focusing solenoid field, strike the waveguide surface and dissipate their kinetic energy as heat.

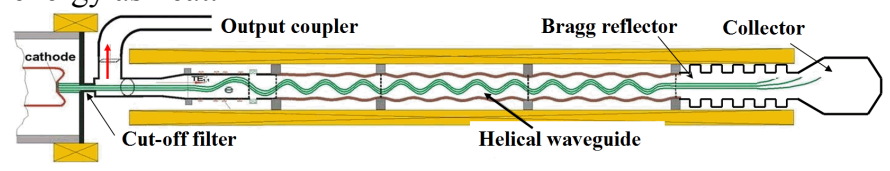

Fig. 1 Gyro-BWO experimental setup.

\section{DESIGN OF THE DEPRESSED COLLECTOR}

Depressed collectors are passive converters that can transfer the kinetic energy of the spent electrons into potential electric energy. "Depressed" means the collector has a depressed potential as compared with the main body of the tube. The electrons lose their kinetic energy when passing through the retarding electrostatic field and finally land on the collector surface with a significant reduction in kinetic energy. They produce a loop current which results in a power recovery from the spent electrons. The power recovered by depressed collectors is defined as [7].

$$
P_{c o l}=\sum_{n} V_{n} I_{n}
$$

Here $n$ is the number of stages and $V_{n}$, In are potentials and collected current on the nth-stage electrode, respectively. By introducing a depressed collector with a collection efficiency of $\eta_{c o l}$, the overall efficiency of the microwave tube $\eta_{t o t}$ with an electronic efficiency $\eta_{e}$ can be calculated using

$$
\eta_{\text {tot }}=\frac{P_{\text {out }}}{P_{b}-P_{\text {col }}}=\frac{\varepsilon_{\text {out }} \eta_{e}}{1-\eta_{\text {col }}\left(1-\eta_{e}\right)}
$$

where $\varepsilon_{\text {out }}$ is the output efficiency defined by the generated microwave power and the output microwave power.

The collection efficiency of a depressed collector is mainly depended on the potentials on the electrodes and the geometry of the electrodes. When specifying a stage number, the optimum potentials on the electrodes can be evaluated by the energy distribution of the spent electron beam which was exported from the simulation of the gyro-BWO using the 3D PIC code MAGIC, as shown in table 1. It was found that when the stage number increased beyond four stages, the collection efficiency did not significantly increase.

Table 6.1 Collection efficiency at different stage number.

\begin{tabular}{|l|c|l|l|l|l|l|c|}
\hline No. & \multicolumn{5}{|c|}{$\begin{array}{c}\text { Potentials on electrodes (kV) } \\
\text { (Comparing with ground voltage) }\end{array}$} & $\begin{array}{c}\text { Col. } \\
\text { Eff. }\end{array}$ \\
\hline 1 & -9.24 & ----- & ----- & ---- & ---- & ---- & $28.8 \%$ \\
\hline 2 & -9.24 & -25.70 & ----- & ----- & ----- & ----- & $63.6 \%$ \\
\hline 3 & -9.24 & -22.14 & -36.57 & ----- & ----- & ----- & $75.7 \%$ \\
\hline 4 & -9.24 & -19.55 & -27.31 & -40.00 & ----- & ----- & $82.5 \%$ \\
\hline 5 & -9.24 & -18.86 & -24.96 & -30.78 & -40.00 & ----- & $85.7 \%$ \\
\hline 6 & -9.24 & -16.98 & -22.14 & -27.22 & -32.66 & -40.00 & $87.5 \%$ \\
\hline
\end{tabular}

Secondary electron emission was applied in the simulation of the depressed collector to investigate the effect of the secondary electrons [8]. Secondary electrons are generally divided into two classes. The first class is the true secondary electrons (TSEs) which are knocked out from the surface of the material due to the bombardment of the primary electrons. The other class is the backscattered electrons (BSEs) including 
the rediffused electrons and the elastic scattering electrons.

In our simulation using the 3-D PIC code MAGIC, the TSE yield function is based on that by Thomas [9]. However, MAGIC also allows users to define their own SEY functions and the energy distribution function for TSEs by using the command "EMISSION SECONDARY." In the simulation, Vaughan's SEY formula [10] was used to evaluate the true secondary yield as it has been proved to be in good agreement with the experimental data.

The scattering process in MAGIC was carried out by ITS (The Integrated TIGER Series of Coupled Electron/Photon Monte Carlo Transport Codes) code, and it has been proven that the simulation results of the ITS code were in good agreement with the experiments [11]. "BACKSCATTER" option in MAGIC allows ITS to be invoked automatically to simulate the emission of both the rediffused and backscattered elastic electrons.

The optimum geometry of the four-stage depressed collector as well as the trajectories of the primary electron, true secondary electrons and the backscattered electrons is shown in Fig. 2.

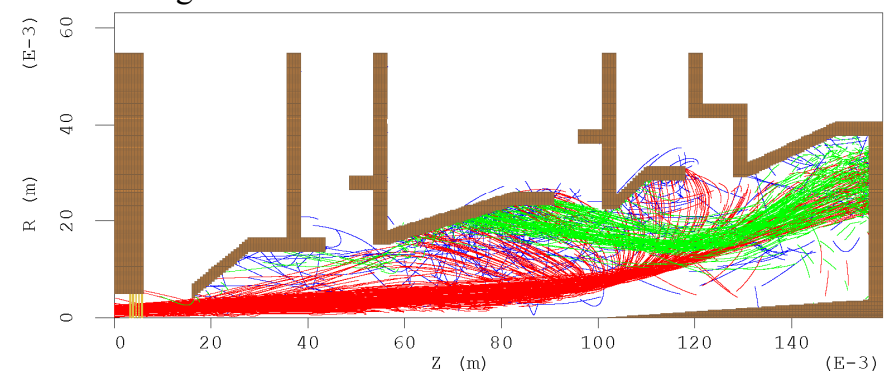

Fig. 2 Trajectories of the electrons (primary electrons in RED, TSEs in GREEN, BSEs in BLUE) in the depressed collector (using Vaughan's formulas).

The collection efficiencies and the backstreaming rate of the W-band Gyro-BWO were therefore simulated in the whole frequency tuning range by the optimized configuration of the designed four-stage depressed collector and are shown in Fig. 3 . The collection efficiencies achieved were simulated to be about $70 \%$ and the backstreaming rate was lower than $7 \%$ in the working frequency band.

The heat dissipation on the electrodes is another important issue in designing the depressed collector. Although the kinetic energy of the spent electrons reduced significantly when they passed through the retarded electric field, the heat due to the power of the remaining kinetic energy of the spent electrons when they struck the surface of the electrodes was still large. The spent electrons with such high kinetic energy could damage the surface of electrodes in the form of a "hot spot" and could reach the thermal stress threshold of the collector material. Fig. 3 shows the maximum heat density and the maximum average heat density in the designed depressed collector when different magnetic fields are applied. The maximum heat density was $\sim 240 \mathrm{~W} / \mathrm{cm}^{2}$. It is lower than the thermal stress threshold of the copper material thus the generation of "hot spots" can be avoided.

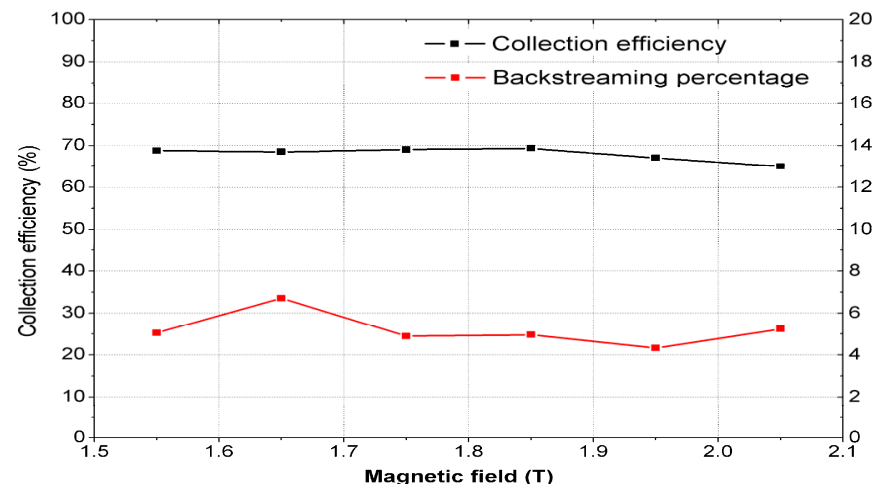

Fig. 3 The collection efficiencies and the backstreaming rate over the frequency tuning range of the Gyro-BWO.

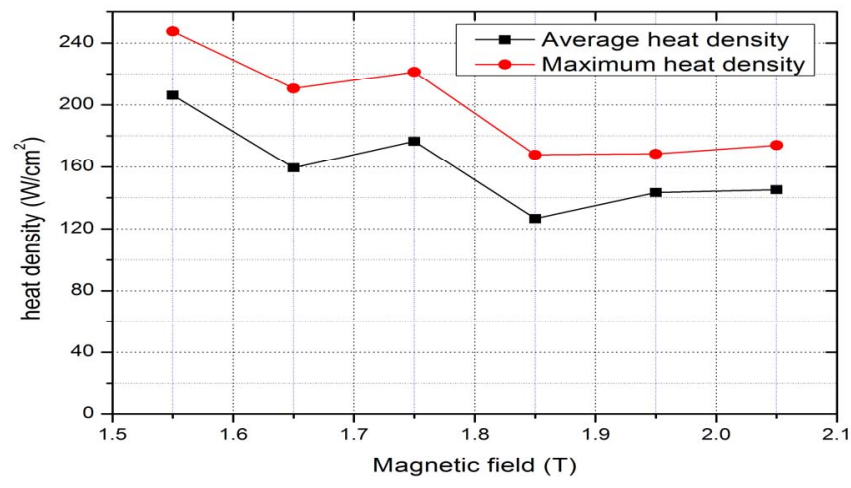

Fig. 4 The maximum heat power density and maximum average power density in different magnetic fields.

\section{REFERENCES}

[1] W. He, K. Ronald, A.R. Young, A.W. Cross, et al, "Gyro-BWO experiments using a Helical Interaction Waveguide", IEEE trans. Electron devices, 52, 839, 2005.

[2] W. He, A.W. Cross, A.D.R. Phelps, K. Ronald, et al, "Experiments and simulations and Gyro-BWOs using a helically corrugated waveguide", Applied Physics Letts, 89 (9): Art. No. 091504, 2006.

[3] W. He, A.D.R. Phelps, C.R. Donaldson, A.W. Cross and K. Ronald, "The Design and Simulation of a W-band Gyro-BWO", Eighth IEEE International Vacuum Electronics Conference, Kitakyushu International Conference Center, Kitakyushu, Japan, May 15-17, 2007.

[4] W. He, C. G. Whyte, E. G. Rafferty, A. W. Cross, et al, "Axis-encircling electron beam generation using a smooth magnetic cusp for gyrodevices”,Appl. Phys. Lett., 93, 121501, 2008.

[5] C.R. Donaldson, W. He, A.W. Cross, A.D. R. Phelps, et al, "Design and Numerical Optimization of a Cusp-Gun-Based Electron Beam for Millimeter-Wave Gyro-Devices”, IEEE Trans. Plasma Sci., 37, No. 11, pp2153, 2009.

[6] C. R. Donaldson, W. He, A. W. Cross, F. Li, et al, "A Cusp Electron Gun for Millimeter Wave Gyro-Devices", Appl. Phys. Lett., 96, 141501, 2010.

[7] L. Zhang, W. He, A. W. Cross, A. D. R. Phelps, et al, "Design of an energy recovery system for a gyrotron backward-wave oscillator", IEEE Trans. Plasma Sci., vol. 37, no. 3, pp. 390-394, Mar. 2009.

[8] L. Zhang, W. He, A. W. Cross, A. D. R. Phelps, et al, "Numerical Optimization of a Multistage Depressed Collector With Secondary Electron Emission for an X-band Gyro-BWO”, IEEE Trans. Plasma Sci., vol. 37 , no. 12, pp. 2328-2334, 2009.

[9] MAGIC User's Manual, Mission Res. Corp., Newington, VA, 2002.

[10] M. Vaughan, "Secondary emission formulas," IEEE Trans. Electron Devices, vol. 40, no. 4, p. 830, Apr. 1993.

[11] J. A. Halbleib, R. P. Kensek, G. D. Valdez, T. A. Mehlhorn, S. M. Seltzer, and M. J. Berger, "ITS: The integrated TIGER series of electron/photon transport codes-Version 3.0," IEEE Trans. Nucl. Sci., vol. 39, no. 4, pp. 1025-1030, Aug. 1992. 